\title{
SEVERIDAD DE LA QUEMAZÓN (Pyricularia oryzae Cav.) EN GERMOPLASMA DE ARROZ F1 EN LA ZONA CENTRAL DEL LITORAL ECUATORIANO
}

\section{SEVERITY OF BURNING (Pyricularia oryzae Cav.) ON F1 RICE GERMPLASM IN THE CENTRAL ZONE IN ECUADORIAN COAST REGION}

\author{
${ }^{\bullet}$ Felipe Rafael Garcés Fiallos ${ }^{1,2}$, Teofilo Gorki Díaz Coronel ${ }^{1,3}$, Ángel Jefferson Aguirre Calderón ${ }^{1}$
}

\author{
${ }^{1}$ Dirección de Investigación Científica y Tecnológica. Universidad Técnica Estatal de Quevedo. Campus finca experimental \\ "La María”, km 7 vía Quevedo-El Empalme. C. P. 73. Mocache, Los Ríos, Ecuador. ${ }^{\circ}$ felipegarces23@yahoo.com \\ ${ }^{2}$ Facultad de Ciencias Agrarias. Universidad Técnica Estatal de Quevedo. Campus Ing. Manuel Haz \\ Álvarez, km 1 1⁄2 vía a Santo Domingo de los Tsáchilas. C. P. 73. Quevedo, Los Ríos, Ecuador. \\ ${ }^{3}$ Facultad de Ciencias Pecuarias. Universidad Técnica Estatal de Quevedo. Campus finca experimental \\ "La María", km 7 vía Quevedo-El Empalme. C. P. 73. Mocache, Los Ríos, Ecuador.
}

\begin{abstract}
Resumen
E $n$ el Ecuador, el cultivo de arroz (Oriza sativa L.) es la principal fuente alimenticia, principalmente formando parte de la dieta básica de los habitantes de la costa ecuatoriana. Entre los factores bióticos adversos al cultivo, está la enfermedad denominada piricularia o quemado del arroz, producida por Pyricularia oryzae Cav., la misma que puede causar daños desde el 59.6\% hasta el 100\%. El objetivo de esta investigación, fue cuantificar la severidad (número de lesiones por hoja) en 39 materiales (líneas y variedades) de arroz de pequeño y mediano porte, divididos en dos experimentos establecidos en la zona central del litoral ecuatoriano. La severidad de piricularia se evaluó en hojas de los estratos inferior, medio y superior de las plantas de arroz, una vez por semana totalizando ocho ocasiones. Se empleó un diseño experimental de bloques completos al azar (DBCA) con 3 repeticiones. Los valores obtenidos fueron integralizados en el Área abajo la curva de progreso de la quemazón del arroz (AACPQA). Para la comparación entre las medias de los tratamientos se empleó la prueba de $\mathrm{T}$ al $5 \%$ de probabilidad de error. Existió comportamiento sanitario diferenciado entre los genotipos evaluados en los dos experimentos. Los materiales sobresalientes fueron la variedad INIAP-16 (9.9), y la línea INIAP-14-7 (11.2) en el primer experimento; mientras que en el segundo fueron la variedad Caluma (17.4) y la línea CC-05-27 (18.8), por haber obtenido una menor AACPQA.
\end{abstract}

Palabras clave: Oriza sativa L., Pyricularia oryzae, número de lesiones por hoja.

Recibido: 27-Febrero-2012. Recibido en forma corregida: 10-Septiembre-2012. Aceptado: 12-Diciembre-2012.

Publicado como ARTÍCULO CIENTÍFICO en Ciencia y Tecnología 5(2): 1-6. 2012

\section{Abstract}

$T$ The rice (Oryzaesativa L.) is the main food source in Ecuador. It is mainly a part of the staple diet of people from coast region. The disease called blast or burning rice caused by Pyricularia oryzae Cav., is among adverse biotic factors to the culture. It can cause damage from $59.6 \%$ to $100 \%$. The objective of this research was to quantify the severity (numbers of lesions per leaf) in 39 materials (lines and varieties) in small and medium size of rice. Both of them were divided into two experiments established in central zone on ecuadorian region. The severity of rice blast was assessed on leaves of the lower, middle and superior strata in plants of rice, once by week, totaling eight times. It was used an experimental design at random complete blocks (RCB) with 3 replications. The values obtained were joined in the Area under progress curve on rice blast (AUPCRB). It was used "T" test at 5\% probability of error for comparison between treatments means. There was differential health behavior between genotypes assessed in both experiments. Outstanding materials in the first experiment were the variety INIAP-16 (9.9) and the lines INIAP-14-7 (11.2) while in the second experiment were the variety Caluma (17.4) and the line CC-05-27 (18.8) for having obtained a least AUPCRB.

Key words: Oriza sativa L., Pyricularia oryzae, number of lesions for leaf. 


\section{INTRODUCCIÓN}

$\mathrm{E}$ Trópico húmedo ecuatoriano y exclusivamente la zona central del litoral, también denominada como cuenca alta del río Guayas, es uno de los lugares agrícolas más importantes a nivel nacional, por su superficie dirigida a la siembra de cultivos transitorios y sus suelos productivos, siendo esta actividad agrícola fuente de ingresos para mucha gente que se dedica directa o indirectamente a la agricultura. En el país, el cultivo de arroz (Oriza sativa L.) es la principal fuente alimenticia, formando parte de la dieta básica de los habitantes de la costa ecuatoriana, según el INEC (2002) reporta un total de 343,936 hectáreas en todo el país, denotando aún más la importancia de esta especie.

Unos de los problemas más serios para este cultivo en cualquier parte del mundo, son los agentes bióticos responsables de las enfermedades (bacterias, espiroplasmas, hongos, protozoarios, micoplasmas, nemátodos $\mathrm{y}$ virus), los mismos que reducen la producción y por ende la rentabilidad del productor dedicado a la siembra de ésta gramínea. El principal problema causado por una enfermedad en todas las regiones arroceras del mundo, es piricularia o quemado del arroz, producida por Pyricularia oryzae Cavara [forma perfecta o sexual: Mangnaporte grisea (T.T. Herbert) Yaegashi \& Udagawa]. Esta enfermedad puede causar daños desde 59.6\% (Prabhu et al., 2003) hasta el 100\% (Filippi et al., 2007), inclusive causando pérdidas de 266,000 toneladas de arroz, como en la India durante el año 2003, representando cerca del 8\% de la producción arrocera de ese país (Salazar-Antón, 2008). Actualmente no se tiene datos de los daños ocasionados por la enfermedad en el Ecuador, a pesar de ser una enfermedad importante en este cultivo. Este hongo transmitido vía semilla, puede según Malavolta et al. (2007) presentar una incidencia de hasta $66.6 \%$.

Ésta es una enfermedad que se encuentra relacionada con los días lluviosos, que desde el punto de vista epidemiológico, favorecen su incidencia en arrozales (Cárdenas et al., 2010), ocurriendo éste clima peculiar en el Trópico húmedo ecuatoriano.

A pesar de que el control químico sea uno de los más utilizados en todo el mundo, desde hace mucho tiempo se viene tratando de minimizar este tipo de control. El uso indiscriminado de productos químicos es un factor que podría reducir la biodiversidad y ocasionar contaminación del ambiente, por lo que sería ideal el empleo principalmente del control biológico de plagas y enfermedades (Bettiol et al., 1997). Por otro lado, la adopción continua del control químico puede acarrear el surgimiento de patógenos resistentes a las diferentes moléculas utilizadas, siendo este hecho mencionado también por Bergamim Filho et al. (1995), Garcés y
Forcelini (2011), Reis et al. (2010) y Zambolim et al. (2007). En el Ecuador es usado el control químico como principal recurso contra la enfermedad.

Como vía alterna a este tipo de control, aparece el genético (resistencia de cultivares a enfermedades). La desventaja más grande de éste control es el tiempo, ya que varios de los programas de mejoramiento genético de cualquier cultivo pueden durar muchos años, e incluso décadas si es el caso de encontrar resistencia poligénica. Sin embargo, Borém y Miranda (2009) relatan que la resistencia a enfermedades constituye uno de los principales objetivos de los programas de mejoramiento de la mayoría de las especies agronómicas y oleícolas. Inclusive López-Tórrez (1995) menciona que la resistencia ejercida por muchas plantas a las enfermedades desempeña un papel importante para el agricultor, el investigador y para el docente del área, lo que denota más todavía la importancia de la investigación en el mejoramiento de las plantas.

En vista de lo mencionado anteriormente, la Dirección de Investigación, Científica y TecnológicaDICYT, perteneciente a la Universidad Técnica Estatal de Quevedo-UTEQ, viene trabajando en el mejoramiento de materiales de arroz, seleccionando materiales genéticos resistentes-tolerantes a la quemazón del arroz, debido a la importancia que suscita la especie en esta región del país. Por lo tanto, el objetivo de ésta investigación fue cuantificar la severidad (número de lesiones por folíolo) en 12 líneas de porte pequeño y 13 de porte mediano F1, y siete variedades, divididos en dos experimentos establecidos en la zona central del litoral ecuatoriano.

\section{MAteriales y MÉTOdos}

T a presente investigación se realizó durante la época lluviosa del año 2011, en el Campus finca experimental "La María", donde se encuentra actualmente la DICYT, perteneciente a la UTEQ, localizada en $79^{\circ} 30^{\prime} 08^{\prime \prime}$ de longitud Oeste y $01^{\circ} 00^{\prime} 35^{\prime \prime}$ de latitud Sur en la zona central del litoral ecuatoriano (73 msnm), siendo esta zona clasificada como bosque húmedo-Tropical (bh-T), con una temperatura promedio de $24.5^{\circ} \mathrm{C}$ y precipitación media anual de $2,178 \mathrm{~mm}$.

La siembra fue realizada el 17 (experimento de los materiales de porte pequeño) y 18 (experimento de los materiales de porte mediano) de enero del 2011, a chorro continuo, a una distancia de $0.25 \mathrm{~m}$ entre hileras, obteniendo una densidad de 70 plantas por metro lineal. Las semillas fueron previamente protegidas con el fungicida carbendazim (ingrediente activo), en una relación de $3 \mathrm{~cm}^{3}$ por cada $\mathrm{kg}$ de semilla. El área de cada una las parcelas fue de $5 \mathrm{~m}^{2}$.

La fertilización en ambos experimentos fue 
realizada con fósforo $\left(69 \mathrm{~kg} \mathrm{ha}^{-1}\right)$ y potasio $(100 \mathrm{~kg}$ $\mathrm{ha}^{-1}$ ) al momento de la siembra, y tres aplicaciones de nitrógeno al 46\% $\left(100 \mathrm{~kg} \mathrm{ha}^{-1}\right)$.

Para el control de plagas fue utilizado methamidophos $\left(0.6 \mathrm{~L} \mathrm{ha}^{-1}\right)$ dos veces, y clorpirifos $\left(0.4 \mathrm{~L} \mathrm{ha}^{-1}\right)$, una vez. También fueron realizadas cuatro deshierbas manuales y dos aplicaciones de paraquat $\left(2.0 \mathrm{~L} \mathrm{ha}^{-1}\right)$ y glifosato $\left(2.0 \mathrm{~L} \mathrm{ha}^{-1}\right)$, para el control de arvenses. No fue aplicado ningún fungicida en la parte aérea para el control de enfermedades.

Se establecieron dos experimentos, siendo el primero conformado por 12 líneas de porte pequeño (CC-05-1, CC-05-11, CC-05-12, Dorado-8, CC-04-7, CC-04-8, CC-04-9, CC-05-2, INIAP-14-3, INIAP-14-4, INIAP-14-7 e INIAP-16-6) y 13 líneas de porte mediano (CC-05-3, CC-05-10, CC-05-22, CC-05-26, CC-05-27, Dorado-3, Caluma-2, CC-03-2, CC-04-1, CC-04-2, CC-05-1, INIAP-16-1 e INIAP-16-4), colocando como testigos absolutos en cada uno de los experimentos, siete variedades CC-03, CC-05, INIAP-14, INIAP-16, F-50, Caluma y CC-04. Las líneas promisorias F1 fueron obtenidas por selección individual.

En estos experimentos fue cuantificada la severidad (número de lesiones por hoja) de la quemazón ( $P$. oryzae), en hojas de plantas de arroz. Esta labor fue realizada semanalmente (24 febrero; 03, 10, 17, 24 y 31 marzo; y 06 y 14 de abril), totalizando ocho evaluaciones. En todas las fechas, fueron recolectadas tres hojas de los estratos inferior, medio y superior (una hoja de cada estrato) de cuatro plantas ubicadas en dos hileras centrales de la parcela de cada tratamiento. Luego, en estas hojas fue contabilizado el número de lesiones mayor o igual a $2 \mathrm{~mm}$ encontrados en la hoja.
El diseño experimental empleado en ambos experimentos, fue el de Bloques Completos al Azar (DBCA) con 3 repeticiones. Los valores obtenidos fueron integralizados en el Área abajo la curva de progreso de la quemazón del arroz (AACPQA), calculado por la ecuación de integración trapezoidal, descrita por Campbell y Madden (1990). Fueron realizados los testes de Bartlett y de Shapiro-Wilks, para verificar si existe homocedasticidad (variancias) y normalidad (residuos), respectivamente. Al constatarse que los datos son homogéneos pero carecen de normalidad, no siendo satisfechos los presupuestos (los datos deben poseer homocedasticidad y normalidad), se procedió a transformar los datos a log (x), luego para la comparación entre las medias de los tratamientos se empleó la prueba $\mathrm{T}$ al $5 \%$ de probabilidad de error. Para todo ésto, se utilizó el software ASSISTAT versión 7.5 beta (2012) desarrollado por Silva y Azevedo (2002).

\section{RESUltados Y DisCuSión}

$\mathrm{L}$ a sintomatología de la quemazón encontrada en las hojas del germoplasma de arroz evaluado en estos experimentos, fueron lesiones de forma romboide y/o elíptica (ancha en el centro y puntiagudas en cualquiera de sus extremos), de coloración castaño. Cuando la enfermedad avanza en el tiempo, el centro de las lesiones presenta oscurecimiento con bordes color café rojizo y/o halo amarillo, hasta tornarse finalmente manchas necróticas. Estas características son descritas en la literatura por Mueller (1984), Webster y Gunnell (1992), Bedendo y Prabhu (2005) y Agrios (2005).

La enfermedad, en ambos experimentos apareció

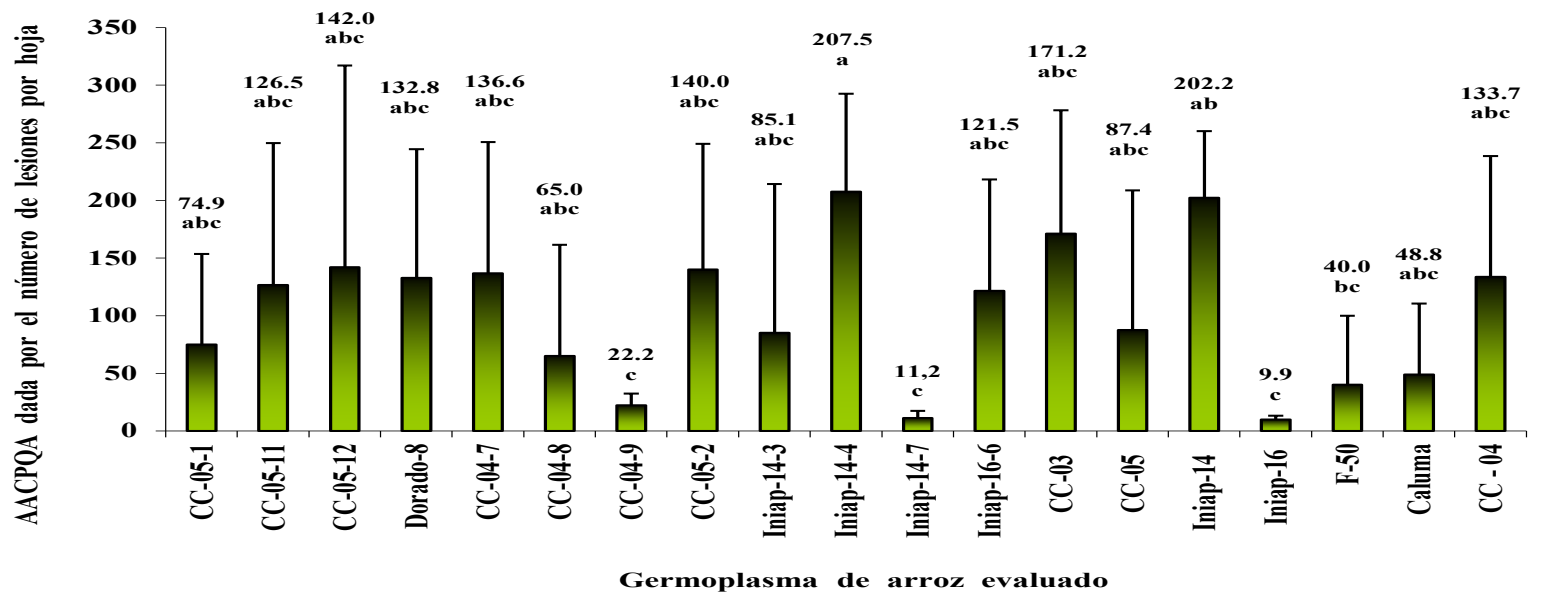

Figura 1. Área abajo la curva de progreso de la quemazón del arroz (AACPQA) dada por el número de lesiones por hoja, cuantificada en 12 líneas promisorias de porte pequeño y 7 variedades comerciales de arroz, durante la época lluviosa del año 2011. Campus finca experimental "La María”, Mocache, Los Ríos, Ecuador. CV: 34.37. DMS: 0.95872 
durante el estadío fenológico vegetativo V13 (collar formado en la hoja 13 o bandera) según Counce et al. (2000), prologándose hasta la etapa final del cultivo. Este hecho concuerda con Bedendo y Prabhu (2005), quienes mencionan que ésta enfermedad se presenta desde los estadíos iniciales del desarrollo hasta la fase final de producción de granos.

En lo referente al Área abajo la curva de progreso de la quemazón del arroz (AACPQA) dada por el número de lesiones por hoja, los valores presentaron diferencias estadísticas entre los materiales. En el primer experimento (Figura 1) se obtuvo valores entre 9.9 (INIAP-16) y 207.5 (INIAP-14-4) unidades, mientras que en el segundo (Figura 2) se alcanzaron valores entre 17.4 (Caluma) y 233.8 (Dorado-3).

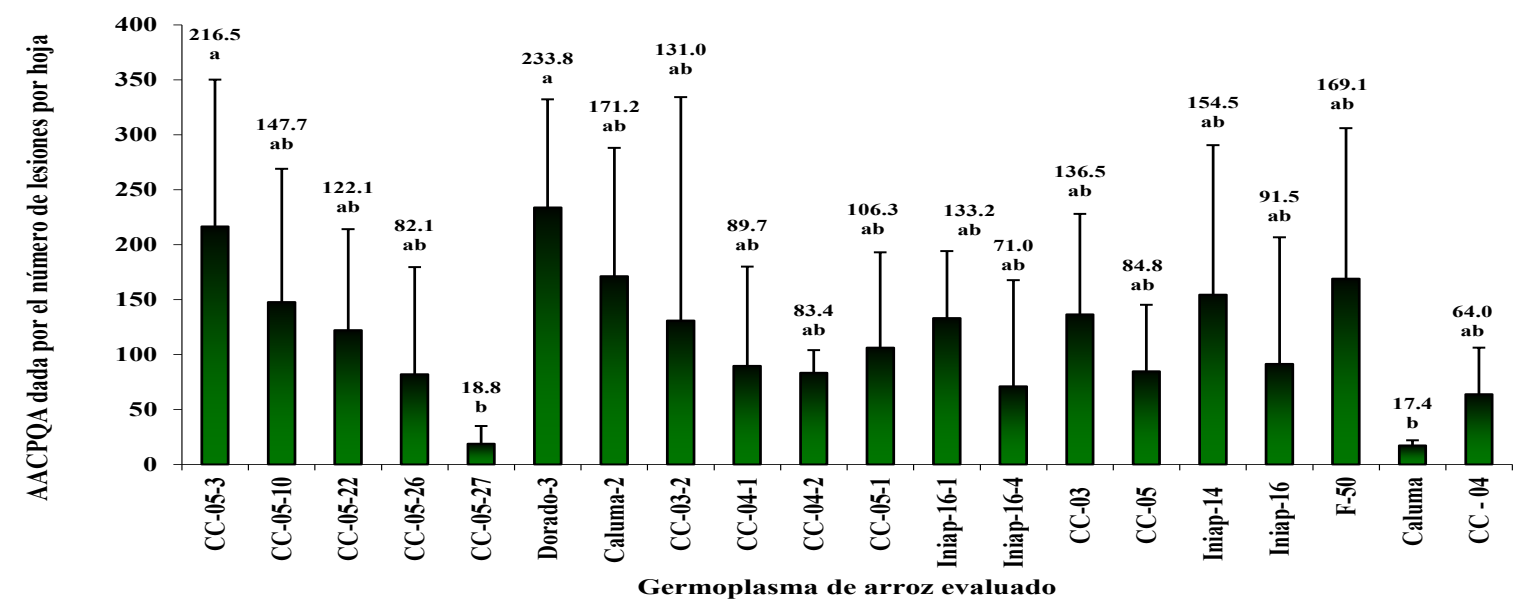

Figura 2. Área abajo la curva de progreso de la quemazón del arroz (AACPQA) dada por el número de lesiones por hoja, cuantificada en 13 líneas promisorias de porte mediano y 7 variedades comerciales de arroz, durante la época lluviosa del año 2011. Campus finca experimental "La María", Mocache, Los Ríos, Ecuador. CV: 28.73. DMS: 0.86778

Los materiales con menores unidades de área y superiores estadísticamente a los demás, fueron la variedad INIAP-16 (9.9), y las líneas INIAP-14-7 (11.2) y CC-04-9 (22.2) en el primer experimento, mientras que en el segundo fueron la variedad Caluma (17.4) y la línea CC-05-27 (18.8).

Los valores encontrados en estos experimentos (primero: 11.2 y 207.5 unidades, y segundo: 17.4 y 233.8 unidades), son inferiores al mínimo (105.2 unidades) y máximo (1,153.0 unidades) obtenidos por Mohapatra et al. (2008) en la India. Estos datos difieren de la presente publicación, por que los autores utilizaron una escala arbitraria de 1 a 5, para la evaluación de la presente enfermedad.

Por otro lado, las diferencias encontradas entre los cultivares estudiados en cada uno de los presentes experimentos, ha sido corroborado por CastejónMuñoz et al. (2007) en España, observando a la cultivar Ullal como la más resistente a $P$. oryzae. Así mismo, en Brasil, Soave et al. (1985) estudiaron 63 genotipos de arroz evaluados en distintos lugares, las cultivares GS-73-164, GS-73-165, GS-73-94, IAC-25 y GS-7317 , aunque susceptibles, presentaron comportamiento satisfactorio a la quemazón del arroz. También en Rumania, Alionte (1997) evaluando algunas enfermedades como Fusarium spp., Helminthosporium spp. y $P$. oryzae, en varios materiales de arroz encontró líneas y cultivares con remarcada resistencia, siendo estos Cristal, Chirnogi, Oltenita, Speranta, F24, F29, F30 y F31. Y por último en Cuba, Echevarría et al. (2010) encontraron nueve materiales genéticos de arroz promisorios que fueron tolerantes a la enfermedad.

Aunque no se pueda definir un comportamiento estable entre estas líneas, por ser materiales segregantes y estar sometidos a un proceso de selección (Cárdenas et al., 2005), este estudio demuestra que existen materiales con características superiores sanitariamente, lo que concordaría con Soave et al. (1985) y Sehly y Bastawisi (1997) quienes mencionan que existe resistencia a piricularia en germoplasma de arroz.

\section{Conclusiones}

Tos materiales sobresalientes en cuanto a su comportamiento a piricularia, fueron la variedad INIAP-16 (9.9), y las líneas INIAP-14-7 (11.2) y CC04-9 (22.2) en el primer experimento, mientras que en el segundo fueron la variedad Caluma (17.4) y la línea CC-05-27 (18.8). 


\section{LITERATURA CITADA}

Agrios, G.N. 2005. Plant Pathology. 5th Ed. Elsevier, Amsterdam. $922 \mathrm{p}$.

Alionte, G. 1997. The incidence of rice diseases in Romanian climate conditions. Cahiers Options Méditerranéennes. 15(3):7-18.

Bedendo, I. P. e A. S. Prabhu. 2005. Doenças do arroz. Em: Kimati, H., L. Amorim, J. A. M. Rezende, A. Bergamin Filho, e L. E. A. Camargo. Manual de Fitopatologia. Vol. 2. 4ta. Ed. pp. 79-90.

Bergamin Filho, A. 1995. Controle genético. Em: Bergamin Filho, A. H. Kimati e L. Amorim, L. (Eds.) Manual de Fitopatologia: Princípios e conceitos. Vol. 1. Ceres, São Paulo, Brasil. pp. 729758.

Bettiol, W., R. Tratch e J. A. H. Galvão. 1997. Controle de doenças de plantas com biofertilizantes. EMBRAPA, Centro Nacional de Pesquisa do Médio Ambiente, Jaguariúma. 2: 22 (Circular Técnica).

Borém, A. e G. V. Miranda. 2009. Melhoramento de Plantas. 5ta. Ed. Universidade Federal de Viçosa, Viçosa. 529 p.

Campbell, C. L. and L. V. Madden. 1990. Introduction to Plant Disease Epidemiology. John Wiley and Sons, New York. 532 p.

Cárdenas, R. M., N. Pérez, E. Cristo, M. C. González y L. Fabré. 2005. Estudio sobre el comportamiento de líneas y variedades de arroz (Oryza sativa L) ante la infección por el hongo Pyricularia grisea Sacc. Cultivos Tropicales 26(4):83-87.

Cárdenas, R. M., S. Mesa, R. Polón, N. Pérez, E. Cristo, L. Fabré y J. J. Hernández. 2010. Relación entre la incidencia de la piriculariosis (Pyricularia grisea Sacc.) del arroz (Oryza sativa Lin.) y diferentes variables climáticas en el complejo agroindustrial arrocero los Palacios. Cultivos Tropicales 31(1):1418.

Castejón-Muñoz, M., I. Lara-Álvarez, and M. Aguilar. 2007. Resistance of rice cultivars to Pyricularia oryzae in Southern Spain. Spanish Journal of Agricultural Research. 5(1):59-66.

Counce, P. A., T. C. Keisling and A. J. Mitchell. 2000. A uniform, objective, and adaptive system for expressing rice development. Crop Science. 40(2):436-443.

Echevarría, A., A. Cruz, N. Pérez, R. M. Cárdenas, D. Rivero y L. Fabré. 2010. Respuesta de 18 líneas de arroz (Oryza sativa L.) ante la piriculariosis en la época poco lluviosa. Cultivos Tropicales 31(2):1720.

Filippi, M. C. C., G. B. Silva e A. S. Prabhu. 2007. Indução de resistência à brusone em folhas de arroz por isolado avirulento de Magnaporthe oryzae. Fitopatologia Brasileira 32:387-392.

Garcés, F. R., y C. A. Forcelini. 2011. Control químico de la roya asiática de la soya en el Planalto Medio, RS., Brasil. Agronomía Mesoamericana 22(2):387395.

INEC. 2002. III Censo Nacional Agropecuario. Resultados Nacionales y Provinciales. Instituto Nacional y Estadística del Ecuador-INEC, Ministerio de Agricultura y Ganadería-MAG, Servicio de Servicio y Censo Agropecuario-SICA. Quito, Ecuador. 255 p.

López-Tórrez, M. 1995. Resistencia de las Plantas. 1ra. Ed. Trillas, México D. F. 103 p.

Malavolta, V. M. A., E. A. Soligo, D. D. Dias, L. E. Azzini e C. R. Bastos. 2007. Incidência de fungos e quantificação de danos em sementes de genótipos de arroz. Summa Phytopathologica 33(3):280-286.

Mohapatra, N. K., A. K. Mukherjee, A. V. Suriya Rao e V. Nayak. 2008. Disease progress curves in the rice blast pathosystem compared with the Logistic and Gompertz models. Journal of Agricultural and Biological Science 3(1):28-37.

Mueller, K. E. 1984. Problemas del Cultivo del Arroz en los Trópicos. International Rice Research InstituteIRRI, Los Baños, Filipinas. 172 p.

Prabhu, A. S., E. M. Castro, L. G. Araujo and R. F. Berni. 2003. Resistance spectra of six elite breeding lines of upland rice to Pyricularia grisea. Pesquisa Agropecuária Brasileira 38:203-210.

Reis, E. M., A. C. Reis e M. A. Carmona. 2010. Manual de Fungicidas. Guia para o controle químico de doenças de plantas. 6ta. Ed. Universidade de Passo Fundo, Passo Fundo. 226 p.

Salazar-Antón, W. 2008. Efecto de factores físicos y bioquímicos sobre la formación de estructuras infectivas de Magnaporthe grisea agente causal de pyriculiarosis en arroz (Oryza sativa). Universitas $1(2): 31-36$.

Sehly, M. R. and A. O. Bastawisi. 1997. The present status of resistance to rice blast disease caused by Pyricularia oryzae under Egyptian conditions. Cahiers Options Méditerranéennes 15(3):87-93.

Silva, F. de A. S. e C. A. V. de. Azevedo, 2002. Versão do programa computacional Assistat para o sistema operacional Windows. Revista Brasileira de Produtos Agroindustriais 4(1):71-78.

Soave, J., L. E. Azzini, de A. O. B. Camargo, A. Pettinelli Junior e M. Sakai. 1985. Identificação de progênies de arroz resistentes à brusone no Estado de São Paulo, em 1976-1977. Bragantia 44(1):311329.

Webster, R. K. and P. S. Gunnell. 1992. Compendium of Rice Diseases. The American Phytopathological 
Garcés et al.

Society-APS, St. Paul. 62 p.

Zambolim, L., W. S. Venâncio, S. H. Furlan de Oliveira. 2007. Manejo de Resistência de Fungos a Fungicidas. Suprema Gráfica e Editora, Visconde do Rio Branco. 168 p. 